\section{Saúde bucal em adultos e idosos na cidade de Rio Claro, São Paulo, Brasil}

\author{
Oral health in adults and the elderly \\ in Rio Claro, São Paulo, Brazil
}

\author{
1 Programa de Pós-Graduação \\ em Odontologia, \\ Faculdade de Odontologia \\ de Piracicaba, Universidade \\ Estadual de Campinas, \\ Piracicaba, Brasil. \\ 2 Departamento de \\ Odontologia Social, \\ Faculdade de Odontologia \\ de Piracicaba, Universidade \\ Estadual de Campinas, \\ Piracicaba, Brasil. \\ Correspondência \\ Maria da Luz Rosário de Sousa \\ Departamento de \\ Odon tologia Social, \\ Faculdade de Odontologia \\ de Piracicaba, Universidade \\ Estadual de Campinas. \\ Av. Limeira 901, Piracicaba, \\ SP 13414-018, Brasil. \\ luzsousa@fop.unicamp.br
}

Débora Dias da Silva 1

Maria da Luz Rosário de Sousa 2

Ronaldo Seichi Wada 2

\section{Introdução}

The aim of this study was to verify the prevalence of dental caries, edentulism, and use of and need for complete dental prostheses in adults and the elderly in Rio Claro, São Paulo State, Brazil. The total sample included 202 subjects, 101 elderly (64 to 75 year-olds) and 101 adults (35 to 44 year-olds), who were examined by four examiners according to who criteria. Edentulism was $74.25 \%$ in the elderly and $8.91 \%$ in adults, and present teeth rates were 3.19 and 22.10 , respectively. In the elderly and adults, DMFT was 31.09 and 22.86, respectively. MT was the major component of the DMFT index in the elderly group (92.64\%), as compared to FT in the adult group (57\%), followed by MT (40.54\%). complete upper and lower dental prostheses were needed by only $1 \%$ of the adults; however, 48.5\% of the elderly required complete upper prostheses and 45.5\% complete lower prostheses. the data suggest that preventive and educational programs are needed for both the elderly group and adults in order for adults to enjoy good oral health when they reach old age.

Adult;Aged; Oral Health; Dental Health Surveys
Tendo em vista o aumento da expectativa de vida tanto em países desenvolvidos como em países em desenvolvimento, torna-se evidente o aumento da população de indivíduos com idade acima de 65 anos.

Com relação aos adultos, embora existam poucos relatos sobre a saúde bucal, estes constituem a larga maioria da população que demanda fortemente por serviços odontológicos e influenciam decisivamente o comportamento de seus dependentes 1 .

A população de Rio Claro é constituída atualmente por 168.218 habitantes, dos quais 8.520 são indivíduos com idade entre 65 e 74 anos e 25.402 entre 35 e 44 anos, o que corresponde respectivamente a $5 \%$ e $16 \%$ do total da população 2 .

A saúde bucal da população idosa brasileira encontra-se numa situação precária, sendo evidenciada em muitos estudos pelo grande número de indivíduos edêntulos 3,4,5,6, como também pela ausência de programas voltados para este grupo populacional 4 .

Nesse contexto, a situação epidemiológica em termos de saúde bucal da população idosa, no Brasil, pode ser classificada atualmente como bastante grave, refletindo o descaso geral com que este grupo é socialmente considerado ${ }^{7}$. As extrações em série, a cárie dentária e a doença periodontal têm tido como conse- 
qüência o edentulismo que resulta num grande número de indivíduos usando próteses totais e/ou delas necessitando.

No Brasil, estudos direcionados para os problemas bucais de idosos e adultos são escassos, e a necessidade de tratamento especificamente para os idosos está geralmente relacionada à perda dos dentes, cárie dentária, abrasão, doença periodontal, câncer bucal e lesões da mucosa bucal 4 .

Em se desconhecendo as reais condições da saúde bucal da população brasileira, não tem havido uma adequada atenção à saúde bucal que abranja todos os grupos populacionais, inclusive adultos e idosos 8 Portanto, o objetivo deste estudo foi verificar a prevalência de cárie, doença periodontal, uso e necessidade de próteses totais e também de edentulismo em idosos e adultos no Município de Rio Claro, para que, por intermédio do conhecimento destas condições, possam ser estabelecidas ações específicas direcionadas a estes grupos.

\section{Material e métodos}

Este é um estudo transversal que fez parte do Levantamento das Condições de Saúde Bucal do Estado de São Paulo 9,10, com a participação de vários municípios pertencentes à DIR XV (Direção Regional de Saúde da região de Piracicaba, que inclui 25 municípios, inclusive Rio Claro). Quatro examinadores foram calibrados de acordo com os critérios da Organização Mundial da Saúde (OMS) para a coleta de dados 11.

Segundo a OMS 11, a faixa etária para estudos epidemiológicos em saúde bucal em indivíduos idosos é de 65 a 74 anos, e em adultos, de 35 a 44 anos. A população de referência foi composta por professores de primeiro grau, funcionários de escolas públicas e particulares de 35 a 44 anos, bem como usuários de serviços de saúde e associados de grêmios de 65 a 74 anos.

Assim, a amostra foi composta por 101 idosos e 101 adultos, na faixa etária entre 65 e 74 e 35 a 44 anos, respectivamente, e houve $10 \%$ de reexames para o cálculo da concordância intraexaminadores.

Para avaliar as condições dos dentes e necessidades de tratamento, foram registrados: coroas e raízes hígidas; cariadas; restauradas, com e sem cáries; ausentes devido à cárie ou por outros motivos; suportes para próteses; coroas protéticas ou facetas/implantes; den- tes não irrompidos; raízes não expostas; traumatismos; excluídos e/ou não registrados, segundo critérios da OMS 11, adaptados pela Faculdade de Saúde Pública da Universidade de São Paulo(FSP/USP) 9,10. Desse modo, o índice para verificar a experiência de cárie foi o CPO-D, que totaliza o número de dentes cariados, restaurados/obturados e perdidos por indivíduo.

A avaliação das condições periodontais foi realizada usando-se o Índice Periodontal Comunitário (CPI), o qual aborda três indicadores das condições periodontais: sangramento gengival, cálculo e bolsas periodontais, em que a cavidade bucal é dividida em sextantes, cada qual com seus dentes índices. Um sextante deveria ser examinado somente se existissem dois ou mais dentes presentes (desde que não estivessem indicados para exodontia). Na ausência dos dentes índices em um sextante qualificado para o exame, todos os dentes remanescentes naquele sextante foram examinados e o índice mais alto registrado.

A perda de inserção periodontal (PIP) foi obtida por exames realizados com base nos dentes indicadores, tendo dessa forma uma estimativa da destruição acumulada durante a vida útil da inserção periodontal. Tanto para avaliação das condições de cárie da coroa (CPOD), quanto para o CPI e PIP, foram usadas sondas milimetradas preconizadas pela OMS e os sextantes nulos (sem pelo menos dois dentes presentes e não indicados para exodontia) foram avaliados nesta análise, como pior condição, ou seja, perda dos dentes.

Com relação à presença (uso) e à necessidade de próteses totais, foi realizado um registro tanto para a maxila quanto para a mandíbula.

Na calibração verificou-se que a porcentagem de concordância interexaminadores, que foi de 97,5 para cárie, 96,1 para PIP, 98,3 para uso e 98,1 para necessidade de próteses, sendo classificadas como excelente concordância, com exceção do CPI, em que a porcentagem de concordância foi de 89,4 , indicando muito boa concordância 12 .

Em se tratando de uma pesquisa que envolve seres humanos, esta se iniciou após a aprovação do comitê de ética em pesquisa (processo no COEP 62/98). 


\section{Resultados}

Do total da amostra, $61,4 \%(n=62)$ dos idosos eram do sexo feminino e $38,6 \%(n=39)$ do masculino; do total dos adultos, $89 \%(\mathrm{n}=90)$ eram do sexo feminino e $11 \%(\mathrm{n}=11)$ do masculino.

Os $10 \%$ de reexames realizados durante a coleta de dados mostraram uma concordância intra-examinadores de 98,4 para cárie; 93,8 para CPI; 98,0 para PIP; e não houve discordância para uso e necessidade de próteses, o que demonstrou a precisão dos resultados encontrados neste estudo, estando de acordo com os relatados por Frias 12.

O CPO-D variou entre 8 e 32 para os adultos e a variação para os idosos foi entre 16 e 32 . Para os idosos, o CPO-D = 32 foi representado por $83,2 \%(n=84)$, e somente $13,9 \%(n=14)$ dos adultos apresentaram esta condição. Torna-se importante ressaltar que a porcentagem de edentulismo foi de $74,25 \%$ e $8,91 \%$, respectivamente.

Os idosos apresentaram CPO-D médio de 31,09 , observando-se um grande número de dentes perdidos $(n=2.909)$, a saber, uma média de 28,80 dentes, o que representou cerca de $92,64 \%$. Já o CPO-D relativo aos adultos foi de 22,86 , apresentando maior porcentagem de dentes restaurados $(57 \%)$, seguida de dentes perdidos (40,54\%) (Tabela 1).

A média de dentes hígidos foi de 0,91 para os idosos e 8,5 para os adultos; quanto aos dentes presentes, a média foi de 3,19 e 22,10 , respectivamente (Tabela 1).

Com relação ao grau periodontal, verificouse que dos 101 idosos, apenas $27 \%(n=27)$ apresentavam pelo menos alguns dentes para avaliação deste índice; com relação aos adultos, tal

Tabela 1

Média de dentes permanentes cariados, obturados/cariados, obturados, perdidos, hígidos, presentes e composição percentual do CPO-D. Rio Claro, São Paulo, Brasil, 1998.

\begin{tabular}{lccrr}
\hline Condição & \multicolumn{2}{c}{ Idosos } & \multicolumn{2}{c}{ Adultos } \\
& Média (DP) & $\%$ & Média (DP) & $\%$ \\
\hline Cariados & 0,50 & 1,62 & 0,37 & 1,60 \\
Obturados/Cariados & 0,00 & 0,00 & 0,20 & 0,87 \\
Obturados & 1,78 & 5,73 & 13,03 & 56,99 \\
Perdidos & 28,80 & 92,64 & 9,27 & 40,54 \\
CPO-D & $31,09(2,47)$ & 100,00 & $22,86(5,65)$ & 100,00 \\
Dentes hígidos & 0,91 & & 8,50 & \\
Dentes presentes & 3,19 & & 22,10 &
\end{tabular}

$\mathrm{DP}=$ desvio padrão. porcentagem foi mais elevada, $92 \%(n=92)$, comprovando que estes apresentam um número maior de dentes.

A condição periodontal com maior prevalência foi periodonto hígido, em que 19 idosos e 73 adultos representaram $70,4 \%$ e $79,3 \%$, seguido de tártaro, em que $25,9 \%$ e $14,1 \%$ possuíam estas condições (Figura 1). Em relação aos sextantes, os idosos apresentaram apenas $11,4 \%$ dos sextantes hígidos e os adultos, $74 \%$.

A perda de inserção com maior freqüência foi entre 0 e $3 \mathrm{~mm}$ com uma porcentagem de $85,2 \%$ para os idosos e $86,8 \%$ para os adultos (Figura 2).

Da amostra de idosos, $52,48 \%(n=53)$ usavam próteses totais superiores e $35,64 \%(n=36)$ inferiores; dentre os adultos, somente $18,81 \%$ $(\mathrm{n}=19)$ e $7,9 \%(\mathrm{n}=8)$ usavam próteses totais superiores e inferiores, respectivamente (Figura 3).

Com relação aos idosos, a necessidade de próteses totais foi de $48,51 \%(\mathrm{n}=49)$ para superior e de $45,54 \%$ ( $n=46)$ para a inferior; para os adultos, esta necessidade foi de apenas $1 \%$ $(\mathrm{n}=1)$, para ambas as arcadas (Figura 4).

\section{Discussão}

Este estudo apresentou limitação em virtude de a amostra de adultos ter sido composta por funcionários das escolas, desta forma estes dados de adultos se tornam preliminares, visto que são escassos na literatura. Note-se que esta foi a abordagem recomendada no Levantamento das Condições de Saúde Bucal do Estado de São Paulo 9,10, possibilitando a comparação com dados de outros locais abordados neste levantamento. Entretanto, os idosos pertenciam a grupos de terceira idade e de serviços, isto é, permitem uma comparação mais ampla com outros estudos.

No trabalho de Silva 4, realizado em Araraquara, em uma população de indivíduos com mais de sessenta anos, o CPO-D foi de 26,66 , composto por $77,19 \%$ de dentes perdidos, sendo a média de dentes presentes de 6,98, não diferindo deste estudo com relação ao CPO-D; todavia, o número de dentes presentes foi maior.

Os resultados do trabalho de Carneiro 5 com idosos institucionalizados na Cidade de São Paulo também não diferiram do presente estudo, pois o CPO-D foi de 30,61 , em que 29,47 corresponderam a dentes perdidos, representando $96,28 \%$, tendo em média 2,5 dentes presentes.

Dados semelhantes apresentaram os idosos de Piracicaba, em que Silva et al. 13 encontra- 
ram $62,3 \%$ com $C P O-D=32$, dos quais $60,65 \%$ eram edêntulos e o CPO-D foi de 29,54, composto por 26,72 de dentes perdidos, eqüivalendo a $90,46 \%$. A média foi de cinco dentes presentes e 2,5 hígidos.

Em um levantamento realizado no ano de 1991 em Hong Kong 14, foram examinados 372 adultos. Nenhum destes apresentou edentulismo e $96 \%$ tinham 21 dentes ou mais. O índice CPO-D foi de 8,7, em que, apesar do maior componente ter sido relativo a dentes perdidos, os resultados mostraram uma melhor condição de saúde bucal, comparando com os dados deste estudo.

É importante ressaltar, neste estudo, a diferença que há comparando-se as médias de dentes hígidos e presentes em adultos e idosos, evidenciando que os idosos apresentaram grande número de dentes perdidos com relação aos adultos.

Dados de um levantamento realizado na China 15 , onde a amostra foi de 1.573 adultos e 1.515 idosos, revelaram que os resultados do CPO-D foram 4,8 e 16,1, respectivamente, fato que também diferiu deste estudo, vale dizer, estes indivíduos apresentaram um número maior de dentes não extraídos (presentes na cavidade bucal) e conseqüentemente melhores condições de saúde bucal, apesar de o maior componente do índice de experiência de cárie ter sido de dentes perdidos.

Comparando-se com o levantamento realizado no Estado de São Paulo 16, tem-se que o CPO-D médio foi de 28,98 e 20,53 para idosos e adultos, respectivamente. A média de dentes perdidos foi de 27,31 e 6,51, demonstrando que há uma igualdade com a população idosa de Rio Claro e evidenciando o problema de predominância de dentes perdidos, aumentando com isto o número de idosos edêntulos e mostrando a real necessidade da existência de programas preventivos para este grupo etário.

Quanto à condição periodontal, os resultados encontrados por Silva et al. 13 foram de $50 \%$ dos idosos apresentando cálculo/tártaro e 100\% com perda de inserção periodontal entre 0 e $3 \mathrm{~mm}$, valores um pouco acima dos encontrados nos idosos de Rio Claro.

Com relação ao uso de prótese total, Frare et al. 17 relataram que $64,6 \%$ dos idosos eram edêntulos e que a maioria usava prótese total superior por motivos estéticos e não usava inferior por apresentar desconforto, mostrando que existe uma deficiência mastigatória, mesmo com o uso de próteses. Nos idosos de Rio Claro, 74,25\% eram edêntulos, e também houve uma maior porcentagem dos que usavam
Figura 1

Porcentagem de indivíduos examinados, segundo o maior grau periodontal (CPI). Rio Claro, São Paulo, Brasil, 1998.

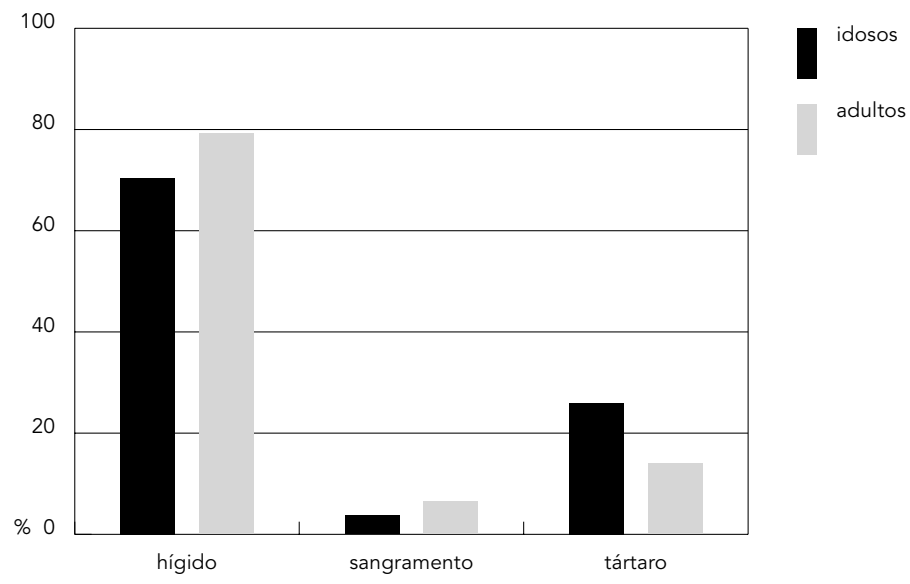

Figura 2

Porcentagem de indivíduos segundo o maior grau de perda de inserção periodontal (PIP). Rio Claro, São Paulo, Brasil, 1998.

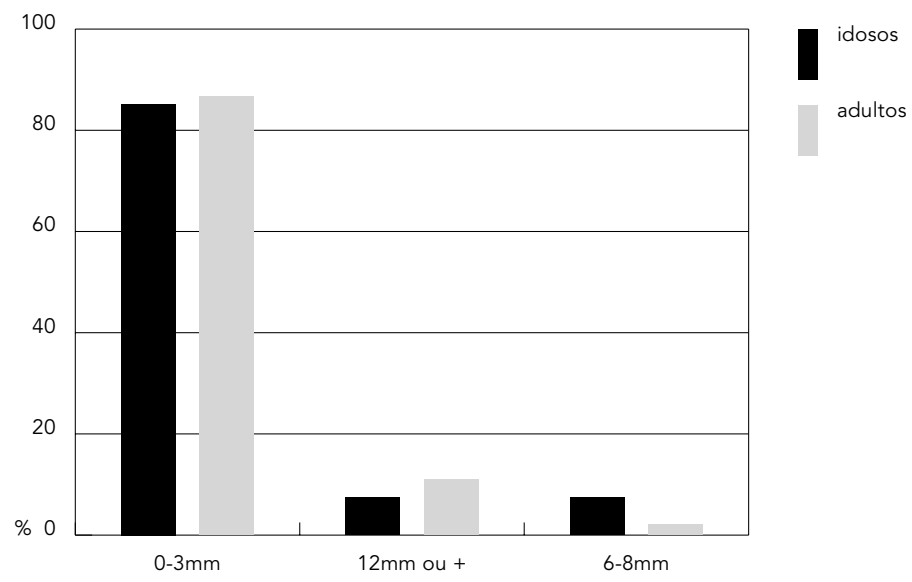


Figura 3

Porcentagem de indivíduos que usam próteses totais (PT).

Rio Claro, São Paulo, Brasil, 1998.

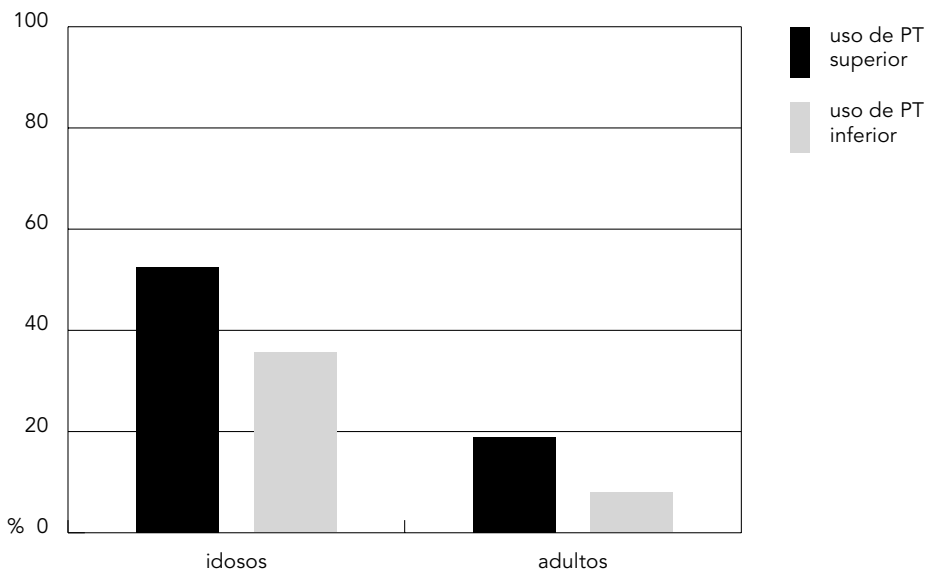

Figura 4

Porcentagem de indivíduos que necessitam de próteses totais (PT).

Rio Claro, São Paulo, Brasil, 1998.

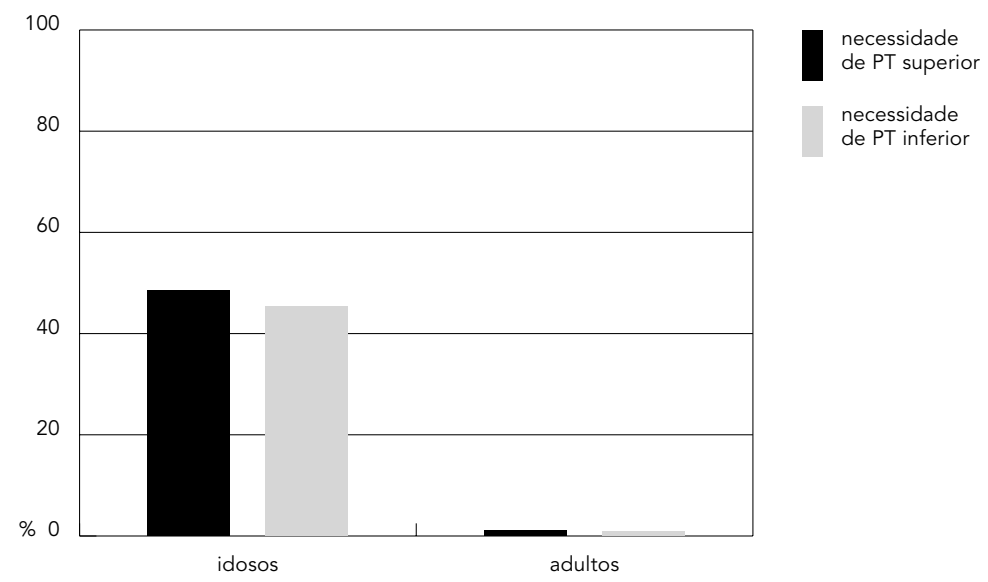

próteses totais superiores comparada com a dos que usavam inferiores.

Quanto ao uso e necessidade de próteses totais, os resultados deste estudo com relação aos idosos, não diferem dos apresentados por Carneiro 5, em que $48,12 \%$ usavam próteses totais superiores e $22,53 \%$ inferiores; e $36,18 \%$ necessitavam de prótese total superior e 50,86\% de inferior

Os dados da população idosa e adulta da DIR XV 10 descrevem que $91 \%$ dos idosos e $5 \%$ dos adultos examinados necessitavam de prótese total superior e apenas $75 \%$ e $3 \%$ de inferior, já os que usavam prótese total, a porcentagem foi de 98 e 50 para a superior e 91 e 33 para a inferior, podendo-se verificar que tanto os idosos como os adultos da DIR XV apresentaram uma porcentagem maior de uso e necessidade de próteses totais.

Diante destes resultados, torna-se importante, mais uma vez, enfatizar que ações de saúde bucal preventivas, curativas e reabilitadoras são necessárias, a fim de que dessa forma possa ocorrer adequadamente a promoção de saúde, com indicações específicas para cada grupo etário.

\section{Conclusões}

Houve uma alta prevalência de edentulismo nos idosos, que apresentaram, em média, apenas um dente hígido (saudável), já as condições de saúde bucal dos adultos difere, apresentando-se em melhores condições.

Recomenda-se dar prioridade à prevenção, para que futuramente possam ser obtidos resultados positivos, ou seja, para que haja uma alta porcentagem de idosos com seus dentes naturais e para que os adultos possam continuar com um número ainda maior de dentes presentes, podendo chegar à terceira idade com condições de saúde bucal melhores do que as encontradas atualmente para este grupo. 


\section{Resumo}

O objetivo deste trabalho foi verificar prevalência de cárie,edentulismo, uso e necessidade de próteses totais em idosos e adultos no Município de Rio Claro, São Paulo, Brasil. A amostra foi de 202 indivíduos, sendo 101 idosos (65 a 74 anos) e 101 adultos (35 a 44 anos). Os exames foram realizados por quatro examinadores, calibrados de acordo com critérios da Organização Mundial da Saúde. A porcentagem de edêntulos foi de $74,25 \%$ para os idosos e $8,91 \%$ para os adultos, sendo a média de dentes presentes de 3,19 e 22,10, respectivamente. O CPOD foi de 31,09 e 22,86 para os idosose adultos, respectivamente. O maior componente do indice CPOD no grupo de idosos foi dentes perdidos (92,64\%);para os adultos, foi dentes restaurados (57\%), seguido de dentes perdidos (40,54\%). Somente $1 \%$ dos adultos necessitava de próteses totais superiores e inferiores, já para os idosos estas necessidades foram de 48,5\% e 45,5\%, respectivamente. Mediante tais resultados, há necessidade de programas preventivos e educativos tanto para idosos como para adultos, a fim de que estes adultos cheguem à terceira idade com uma condição de saúde bucal melhor que a relatada atualmente.

Adulto; Idosos; Saúde Bucal;Levantamentos de Saúde Bucal

\section{Referências}

1. Pinto VG. Saúde bucal para adultos. In: Pinto VG, organizador. Saúde bucal coletiva. São Paulo: Editora Santos; 2000. p. 114-6.

2. Fundação Instituto Brasileiro de Geografia e Estatística. Características da população e dos domicílios. Resultados do universo. Rio de Janeiro: Fundação Instituto Brasileiro de Geografia e Estatística; 2000.

3. Saliba CA, Saliba NA, Marcelino G, Moimas SAS. Saúde bucal dos idosos: uma realidade ignorada. Rev Assoc Paul Cir Dent 1999; 53:279-82.

4. Silva SRC. Autopercepção das condições bucais em pessoas com 60 anos e mais de idade [Tese de Doutorado]. São Paulo: Faculdade de Saúde Pública, Universidade de São Paulo; 1999.

5. Carneiro RMV. Saúde bucal em idosos institucionalizados na Cidade de São Paulo: estudo epidemiológico e de autopercepção [Dissertação de Mestrado]. São Paulo: Faculdade de Saúde Pública, Universidade de São Paulo; 2001.

6. Pinheiro C. Soluções para quem perdeu (todos) os dentes. Rev Assoc Paul Cir Dent 2001; 55:73-81.

7. Jitomirski F. Atenção a idosos. In: Pinto VG, organizador. Saúde bucal coletiva. São Paulo: Editora Santos; 2000. p. 120-7.

8. Shinkai RSA, Cury AADB. O papel da odontologia na equipe interdisciplinar: contribuindo para a atenção integral ao idoso. Cad Saúde Pública 2000; 16:1099-109.

9. Secretaria de Estado da Saúde de São Paulo. Levantamento das condições de saúde bucal - Estado de São Paulo, 1998: Caderno de instruções. São Paulo: Secretaria de Estado da Saúde de São Paulo; 1998.

\section{Colaboradores}

D. D. Silva contribuiu com a idéia original do tema, análise e discussão dos resultados, bem como redação final do artigo. M. L. R. Sousa coordenou o trabalho e elaborou a introdução e as conclusões. R. S. Wada participou do planejamento do trabalho, análise dos resultados e elaboração das conclusões.

10. Secretaria de Estado de Saúde de São Paulo. Condições de saúde bucal: Estado de São Paulo, Direção Regional de Saúde de Piracicaba - DIR XV, 1998. São Paulo: Secretaria de Estado da Saúde de São Paulo; 1998.

11. World Health Organization. Oral health surveys: basic methods. Geneva: World Health Organization; 1997.

12. Frias AC. Estudo de confiabilidade do levantamento epidemiológico de saúde bucal, Estado de São Paulo, 1998 [Dissertação de Mestrado]. São Paulo: Faculdade de Saúde Pública, Universidade de São Paulo; 2000.

13. Silva DD, Sousa MLR, Toledo R, Lisboa CM, Taglietta MF. Condições de saúde bucal em idosos na Cidade de Piracicaba. Rev Assoc Paul Cir Dent 2000; 56:183-7.

14. Lo ECM, Schwarz E. Tooth and root conditions in the middle-age and the elderly in Hong Kong. Community Dent Oral Epidemiol 1994; 22:381-5.

15. Lin HC, Wong MCM, Zhang HG, Lo ECM, Scharwz E. Coronal and root caries in Southern Chinese adults. J Dent Res 2001; 80:1475-9.

16. Secretaria de Estado da Saúde de São Paulo. Levantamento epidemiológico em saúde bucal: Estado de São Paulo, 1998. São Paulo: Secretaria de Estado da Saúde de São Paulo; 1999.

17. Frare SM, Limas PA, Albarello FJ, Pedot G, Régio RAS. Terceira idade: quais os problemas orais existentes? Rev Assoc Paul Cir Dent 1997; 51:573-6.

Recebido em 28/Abr/2003

Versão final reapresentada em 21/Ago/2003

Aprovado em 13/Jan/2004 